\title{
Perfil de mulheres usuárias de álcool em ambulatório especializado em dependência química
}

\author{
Profile of female alcohol users in specialized outpatient clinic \\ Márcia Fonsi Elbreder', Ronaldo Laranjeira¹,2, Marluce Miguel de Siqueira³, Dulce Aparecida Barbosa ${ }^{4}$
}

\section{RESUMO}

Embora cresça o número de mulheres com abuso ou dependência alcoólica, elas ainda permanecem como alvo não prioritário na tomada de decisão dos gestores de políticas públicas. Objetivos: Caracterizar o perfil sociodemográfico de mulheres com abuso ou dependência do álcool, identificar o consumo alcoólico, as intervenções terapêuticas realizadas e alguns fatores que poderiam estar relacionados ao abandono precoce do tratamento nesta população. Metódos: Foram incluídas 192 mulheres que procuraram pela primeira vez tratamento na Unidade de Pesquisa em Álcool e Drogas (UNIAD) da Universidade Federal de São Paulo (UNIFESP), no período de 2000 a 2006. As informações foram coletadas nos prontuários das pacientes, as quais foram separadas em dois grupos, usando-se como critério o abandono de tratamento. Resultados: Não houve diferença significativa nas características sociodemográficas da população estudada. Em ambos os grupos houve predomínio de solteiras, com primeiro grau incompleto e situação de desemprego. O consumo diário de destilados foi significantemente maior no grupo abandono gradual $(p<0,002)$. O grau de dependência grave foi significantemente maior $(p<0,001)$ nos dois grupos em relação aos graus leve e moderado. A quantidade de álcool ingerida por semana, o uso de medicação coadjuvante e a necessidade de atendimento psiquiátrico prévio foi significantemente maior no grupo abandono gradual ( $p<0,001$ e $p<0,002$ ), respectivamente. Conclusão: Mulheres usuárias de álcool que desistiram do tratamento no primeiro mês quando comparadas às não desistentes fizeram mais uso de fermentados ou a associação deste com destilados, consumiram menos unidades de álcool por semana, usaram menos medicações coadjuvantes e procuraram menos tratamentos prévios.

\begin{abstract}
Although the number of women who use, abuse of and become addicted to alcohol is increasing, they are still not the main target of the health care services as their distinctive characteristics regarding psychoactive substances have not been taken into account by the public health policy decision-makers. Objective: To characterise those women who use alcohol as well as to identify
\end{abstract}

Unidade de Pesquisa em Álcool e Drogas (UNIAD) da Universidade Federal de São Paulo (UNIFESP).

1 UNIAD da UNIFESP.

2 Departamento de Psiquiatria da UNIFESP.

Recebido

3/10/2007

Aprovado

3 Núcleo de Educação Aberta e a Distância (NEAD) da Universidade Federal do Espírito Santo (UFES)

4 Programa de Pós-graduação em Educação (PPGE) da UNIFESP.

Endereço para correspondência: Márcia Fonsi Elbreder

Rua Botucatu, 390 - 04023-061 - Vila Clementino, São Paulo, SP

E-mail:mcfonsi@uol.com.br 


\section{Key-words}

Women, alcoholism, drop-out. the pattern of alcohol consumption, clinical interventions, and previous treatments performed in this patient population. Methods: This retrospective cohort study reviewed medical records of alcohol users who had sought treatments offered by UNIAD and UNIFESP between 2000 - 2006. A total of 192 patients were included in this study according to criteria for treatment dropout.

Results: The groups were found to have similar socio-demographic characteristics. Consumption of both fermented and distilled beverages were significantly higher in the dropout group $(p=0.002)$. Alcohol addiction was found to be significantly more severe in both groups $(p<0.001)$. Use of auxiliary medication $(p<0.001)$ and search for psychiatric consultation $(p<0.001)$ were significantly higher in the non-dropout group. Conclusion: Alcoholic women who abandoned the treatment during the first month, compared to those who did not, made more use of both fermented and distilled beverages, had consumed less units per week, used less auxiliary medications, and sought less treatments.

\section{INTRODUÇÃO}

O álcool é uma substância psicoativa que tem seu consumo liberado e incentivado pela sociedade. A mortalidade e a limitação funcional causadas pelo consumo de bebidas alcoólicas superam aquelas ocasionadas pelo tabaco; determinando 3,2\% da mortalidade global, sendo 5,6\% deste valor para homens e 0,6\% em mulheres'.

Na Suécia, cerca de $25 \%$ dos óbitos de menores de 50 anos foram atribuídos ao álcool². Um outro estudo em Lübeck, Alemanha, revelou que idosos acima de 64 anos que deram entrada em hospitais foram diagnosticados como dependentes em $17,7 \%$ do sexo masculino e $4,2 \%$ do sexo feminino ${ }^{3}$.

Estudo sobre mortalidade por dependência de álcool no Brasil (1998-2002) utilizando-se a causa básica de morte das estatísticas oficiais, usando-se como critérios o CID-10 (F10), relata que em 2002 ocorreram 4.580 óbitos masculinos e 515 femininos por dependência de álcool, correspondendo a 0,8\% das mortes masculinas e $0,1 \%$ das femininas ${ }^{4}$.

\section{Mulheres e o álcool}

Existem diferenças no consumo de álcool relacionadas ao gênero, em que o uso abusivo é mais freqüente entre homens, no entanto, existem outros estudos apontando o aumento entre mulheres e de maneira mais precoce, mostrando que a diferença entre os gêneros tem diminuído progressivamente, bem como para outros grupos especiais ${ }^{5}$.

A metabolização do álcool e de outras substâncias psicoativas ocorrem de maneira mais lenta nas mulheres do que nos homens e, assim, elas se tornam mais susceptíveis aos prejuízos associados ao seu consumo, mesmo ingerindo níveis mais baixos de álcool por período mais curto ${ }^{6}$. Comorbidades psiquiátricas associadas ao alcoolismo feminino aparecem com freqüência na literatura, como transtorno de personalidade ${ }^{7}$, depressão ${ }^{8}$, transtorno bipolar ${ }^{9}$, transtornos de ansiedade ${ }^{10}$ e transtornos alimentares ${ }^{11}$.

A despeito de sua emancipação e engajamento em diversas áreas de atuação, as mulheres que consomem substâncias psicoativas, tendem, por causa do preconceito e da estigmatização, a permanecerem anônimas, retardando a procura por ajuda'2.

\section{Abandono de tratamento}

É nítido que qualquer programa de tratamento só surtirá efeito se o paciente permanecer nele o tempo suficiente para que haja a chance de um impacto ${ }^{13}$. Greenfield et al.14, em uma revisão literária, acharam estudos inconsistentes para o abandono de tratamento, pois as mulheres permaneceram durante algumas décadas sub-representadas nas pesquisas. As faltas às consultas podem ser usadas como termômetro da adesão terapêutica ${ }^{15}$.

Corroborando com as observações de Rennó Jr. et al! ${ }^{16}$, há no Brasil carência de serviços especializados no atendimento da saúde mental da mulher, pouco treinamento dos profissionais da saúde e escassez na produtividade de pesquisas. Como compreendemos e vivenciamos no cotidiano profissional, na Unidade de Pesquisa em Álcool e Drogas (UNIAD) da Universidade Federal de São Paulo (UNIFESP), o problema das mulheres alcoolistas que requerem abordagem diferenciada e personalizada e acreditamos que se mais bem conhecermos esta população de pacientes, pode-se estabelecer e implementar medidas terapêuticas mais ajustadas às suas necessidades.

Diante disso, realizamos este estudo que tem como objetivos: caracterizar o perfil sociodemográfico de mulheres com abuso ou dependência do álcool; identificar o tipo e o consumo alcoólico, as intervenções terapêuticas realizadas e alguns fatores que poderiam estar relacionados ao abandono precoce do tratamento. 


\section{MÉTODOS}

\section{Aspectos éticos}

Este estudo foi precedido da aprovação do Comitê de Ética em Pesquisa da UNIFESP.

\section{Desenho, período e local do estudo}

Trata-se de um estudo de coorte, retrospectivo, com busca ativa nos prontuários das pacientes do sexo feminino que apresentavam abuso ou dependência do álcool, segundo critérios da CID-10, que procuraram tratamento pela primeira vez na UNIAD, vinculada ao Departamento de Psiquiatria da UNIFESP, entre 2000 e 2006.

\section{População do estudo}

A amostra englobou 192 pacientes, que preencheram os critérios de inclusão. As pacientes foram subdivididas em dois grupos, usando-se como critério o abandono de tratamento. Segundo Baekland e Lundwall' ${ }^{17}$, aquelas que permaneceram em tratamento até o primeiro mês (entre triagem até a quarta consulta) foram incluídas no grupo de "abandono rápido" ( $n=101)$ e as que abandonaram o tratamento após este período (cinco consultas ou mais) foram incluídas no grupo "abandono gradual" $(n=91)$ de tratamento.

\section{Critérios de inclusão}

Mulheres que procuraram pela primeira vez tratamento na UNIAD, no período entre 2000 e 2006 e que preencheram critérios para abuso/uso nocivo ou dependência do álcool, segundo critérios da CID-10.

Mulheres usuárias de outras substâncias psicoativas, sendo o álcool a substância primária.

Comparecimento semanal às consultas.

\section{Coleta de dados}

Realizada por meio de busca ativa nos prontuários das pacientes do sexo feminino. As pacientes do estudo eram oriundas de serviços dentro da própria instituição, de órgãos judiciais, de outros serviços ou chegaram por meio de divulgação da mídia. A marcação da triagem de mulheres sempre foi feita de maneira rápida, em razão da baixa demanda e tentando-se aproveitar o momento em que ela estivesse motivada para o tratamento ${ }^{18}$. As enfermeiras ocuparam-se da triagem e posteriormente da coordenação do grupo específico, pensando-se na questão da identificação do gênero, visando facilitar o vínculo.

A entrevista teria de ser diretiva, clara e flexível, neste momento, confrontos deveriam ser evitados. A meta era registrar os dados sociodemográficos; investigar o padrão e a freqüência do consumo alcoólico; indagar sobre as intervenções terapêuticas prévias e atuais; fazer o diagnóstico diferencial entre abuso e dependência de álcool, segundo a
CID-10; levantar os problemas físicos, psicológicos e sociais decorrentes do uso do álcool; observar os sinais e os sintomas físicos da síndrome de abstinência do álcool (SAA); verificar a necessidade de desintoxicação alcoólica (DST) com medicamentos específicos; e aplicar o Short Alcohol Dependence Data (SADD), que afere a presença e a gravidade da dependência do álcool. Este questionário foi rotineiramente aplicado nas usuárias do serviço e foi validado para uso na população brasileira por Jorge e Masur ${ }^{19}$. Constituído por 15 perguntas com quatro opções de respostas: "nunca", "poucas vezes", "muitas vezes" e "sempre", com valores de 0, 1, 2 e 3 pontos, respectivamente, que, de acordo com a soma dos pontos, o paciente pode ser classificado quanto ao grau de dependência: leve (1-9 pontos), moderado (10-19 pontos) e grave (20-45 pontos).

A desintoxicação alcoólica ambulatorial visa a tratar e monitorar a SAA, estabilizando as funções vitais do indivíduo. É um método seguro e simples, mas que exige acompanhamento cuidadoso, pois cada paciente apresenta sintomas distintos e encontra-se em diferentes graus de dependência; podemos dizer que este processo é individualizado e personalizado ${ }^{20}$. Na UNIAD, as enfermeiras desempenhavam essa tarefa, monitoravam as pacientes quanto à evolução dos sinais e dos sintomas da SAA, oferecendo segurança e tranqüilidade às pacientes, esclareciam sobre sua doença e participavam e estimulavam o processo de mudança e sua continuidade no tratamento.

Posteriormente, elas eram encaminhadas ao atendimento com psicólogos, visto que o número de sessões dependia da necessidade individual de cada paciente. Muitas desistiram do tratamento nas primeiras semanas, assim não houve tempo hábil de se agendar consulta médica para a detecção de comorbidade psiquiátrica, bem como solicitar os exames laboratoriais necessários.

Concomitante ao tratamento grupal, as pacientes poderiam manter-se em acompanhamento psicológico e médico. Ainda contávamos com um grupo de família para apoio e suporte aos seus membros.

Na UNIAD, o tratamento é feito quase exclusivamente em nível ambulatorial, que se tem mostrado eficaz na maioria dos casos. Limitamo-nos a internação hospitalar às pacientes com transtornos psiquiátricos e/ou clínicos associados, como hepatopatia, gravidez, risco de suicídio, em caso de tratamento ambulatorial sem resposta ou falta de suporte social. O tratamento é passível de ajustes e deve ser estruturado e planejado de acordo com as particularidades e necessidades de cada paciente, dessa forma, podese dizer que o tratamento é individualizado. As pacientes são orientadas que a meta do tratamento é a abstinência total do álcool. Trabalha-se durante o tratamento questões relacionadas a fatores de risco e proteção, doenças relacionadas ao alcoolismo, conceitos de SDA, SAA, estágios de 
motivação, estratégias para prevenção de recaídas e questões familiares. Foca-se a melhora da auto-estima, visando fortalecimento interno para encarar a realidade externa.

\section{Análise estatística}

Para comparar os grupos, foi utilizado o teste qui-quadrado ou o teste exato de Fisher, quando necessário. Foi considerado $\mathrm{p}<0,05$ para a significância estatística.

\section{RESULTADOS}

Os dados sociodemográficos das mulheres usuárias de álcool estão apresentados na Tabela 1. Não houve diferença significativa estatística quanto às características sociodemográficas, em ambos os grupos. Houve predomínio da situação de desemprego, de mulheres solteiras e predomínio de 1 o grau incompleto, para ambos os grupos. A média de idade foi similar em ambos os grupos. As do grupo "abandono rápido" tinham 40 anos ( $D P=11,7)$ e as do grupo "abandono gradual" tinham 42 anos (DP $=11,1$ ).

Na Tabela 2, as informações relacionadas à história do consumo alcoólico.

Embora não tenha sido encontrada significância estatística em relação à freqüência do consumo alcoólico ( $p=0,389$ ), observamos que houve predomínio em ambos os grupos do uso diário de bebidas. O consumo de bebidas entre cinco a seis vezes por semana foi mais freqüente no grupo "abandono gradual" e de três a quatro vezes por semana foi mais freqüente no grupo "abandono rápido".

Em relação ao tipo de bebida utilizada houve significância estatística $(p=0,002)$ e observamos que o grupo "abandono gradual" consumiu mais bebidas destiladas que o grupo do "abandono rápido", que por sua vez consumiu mais fermentados ou a associação de destilados e fermentados. O grau de dependência grave foi significativamente
Tabela 1. Distribuição de variáveis sociodemográficas da população estudada

\begin{tabular}{|c|c|c|c|c|c|c|c|}
\hline & $\begin{array}{c}\text { Abandono } \\
\text { rápido }\end{array}$ & $(\%)$ & $\begin{array}{c}\text { Abandono } \\
\text { gradual }\end{array}$ & $(\%)$ & Total & $(\%)$ & $p$ \\
\hline Estado Civil & & & & & & & 0,890 \\
\hline Solteira & 35 & $(35)$ & 36 & $(40)$ & 71 & (37) & \\
\hline Casada & 27 & $(27)$ & 22 & $(24)$ & 49 & $(26)$ & \\
\hline Divorciada/desquitada & 17 & (17) & 16 & $(18)$ & 33 & (17) & \\
\hline Viúva & 8 & (8) & 8 & (9) & 16 & (8) & \\
\hline Amasiada & 14 & (14) & 9 & $(10)$ & 23 & $(12)$ & \\
\hline Escolaridade & & & & & & & 0,802 \\
\hline Analfabeto & 8 & (8) & 6 & (7) & 14 & (7) & \\
\hline $1^{\circ} \mathrm{grau}$ incompleto & 38 & (38) & 35 & $(38)$ & 73 & (38) & \\
\hline $1^{\circ}$ grau completo & 3 & (3) & 7 & (8) & 10 & $(5)$ & \\
\hline $2^{\circ}$ grau incompleto & 8 & (8) & 8 & (9) & 16 & (8) & \\
\hline $2^{\circ}$ grau completo & 12 & $(12)$ & 12 & (13) & 24 & (13) & \\
\hline $3^{\circ} \mathrm{grau}$ incompleto & 13 & (13) & 8 & (9) & 21 & (11) & \\
\hline $3^{\circ}$ grau completo & 18 & (18) & 15 & $(16)$ & 33 & (17) & \\
\hline Raça & & & & & & & 0,102 \\
\hline Branca & 72 & (71) & 64 & $(70)$ & 136 & (71) & \\
\hline Negra & 11 & $(11)$ & 3 & (3) & 14 & $(7)$ & \\
\hline Mulata & 17 & (17) & 23 & $(25)$ & 40 & $(21)$ & \\
\hline Amarela & 1 & (1) & 1 & (1) & 2 & (1) & \\
\hline Situação de trabalho & & & & & & & 0,332 \\
\hline Desempregada & 44 & $(44)$ & 41 & $(45)$ & 85 & $(44)$ & \\
\hline Registrada & 17 & $(17)$ & 22 & $(24)$ & 39 & $(20)$ & \\
\hline Autônoma & 13 & (13) & 4 & (4) & 17 & (9) & \\
\hline "Bico"/temporário & 5 & (5) & 8 & (9) & 13 & (7) & \\
\hline Aposentada & 6 & $(6)$ & 5 & (5) & 11 & $(6)$ & \\
\hline Licença médica & 2 & $(2)$ & 2 & (2) & 4 & $(2)$ & \\
\hline Outros & 14 & (14) & 9 & $(10)$ & 23 & $(12)$ & \\
\hline
\end{tabular}

maior ( $p<0,001$ ), em relação aos graus moderado e leve, em ambos os grupos.

A quantidade de álcool ingerida semanalmente foi mais elevada no grupo "abandono gradual" (média $=137,5$ $\mathrm{U} /$ semana, $\mathrm{DP}=112,1)$ se comparadas ao grupo "abandono rápido" (média $=94,4 \mathrm{U} /$ semana, $\mathrm{DP}=77,2$ ) e a idade para o início do consumo regular foi similar para ambos os grupos,

Tabela 2. Distribuição das variáveis relacionadas ao consumo alcoólico, segundo a desistência de mulheres usuárias de álcool no primeiro mês de tratamento

\begin{tabular}{|c|c|c|c|c|c|c|c|}
\hline Variáveis & Abandono rápido & $(\%)$ & Abandono gradual & $(\%)$ & Total & $(\%)$ & $p$ \\
\hline \multicolumn{8}{|c|}{ Freqüência de consumo alcoólico no último mês } \\
\hline Uso diário & 47 & $(47)$ & 42 & $(46)$ & 89 & $(46)$ & 0,389 \\
\hline 5 a 6 dias por semana & 18 & $(18)$ & 27 & $(30)$ & 45 & $(23)$ & \\
\hline 3 a 4 dias por semana & 22 & $(22)$ & 14 & $(15)$ & 36 & (19) & \\
\hline 1 a 2 dias por semana & 8 & (8) & 5 & $(5)$ & 13 & $(7)$ & \\
\hline 2 a 3 dias por mês & 3 & (3) & 2 & $(2)$ & 5 & (3) & \\
\hline 1 vez por mês & 1 & (1) & 1 & (1) & 2 & (1) & \\
\hline$<1$ vez por mês & 2 & $(2)$ & & & 2 & (1) & \\
\hline \multicolumn{8}{|l|}{ Tipo de bebida } \\
\hline Destilado & 32 & $(32)$ & 52 & $(57)$ & 84 & (44) & 0,002 \\
\hline Fermentado & 37 & $(37)$ & 23 & $(25)$ & 60 & (31) & \\
\hline Destilado + fermentado & 32 & $(32)$ & 16 & $(18)$ & 48 & $(25)$ & \\
\hline \multicolumn{8}{|l|}{ Grau de dependência SADD } \\
\hline Leve & 7 & (7) & 2 & (2) & 9 & (5) & $<0,001$ \\
\hline Moderado & 34 & (34) & 12 & (13) & 46 & $(24)$ & \\
\hline Grave & 60 & (59) & 77 & (85) & 137 & (71) & \\
\hline
\end{tabular}


22,6 versus 24,7 anos, respectivamente "abandono rápido" e "abandono gradual".

Na Tabela 3, apresentamos as intervenções terapêuticas às quais as mulheres alcoolistas foram submetidas. O uso de medicação coadjuvante (dissulfiram ou naltrexona) foi significativamente maior no grupo "abandono gradual" ( $p<0,001)$ e ter recebido atendimento com médico psiquiatra foi uma variável com significância estatística ( $p<0,001)$ para a continuidade do tratamento. A busca pelo tratamento prévio foi significantemente menor ( $p=0,028$ ) no grupo "abandono rápido". Quanto ao número de internações psiquiátricas prévias $(\mathrm{N}=1)$, houve similaridade em ambos os grupos, com DP = 2,1 e DP = 1,5 para o grupo "abandono rápido" e "abandono gradual", respectivamente.

O abandono de tratamento da amostra foi de 53,1\% (102 pacientes) já no primeiro mês de tratamento, 13\% no segundo mês (25 pacientes) e 6,8\% (13 pacientes) no terceiro mês.

\section{DISCUSSÃO}

As realidades internacional e nacional nos estudos sobre dependência química mostra que estes estão quase exclusivamente voltados para a população masculina, banalizandose a questão do gênero, e que a compreensão da dinâmica feminina diante deste problema é ainda incipiente.

No Brasil, existem poucos estudos relacionados ao beber, carência de serviços especializados para atendimento desta população, bem como para a formação de profissionais de saúde, modelos de tratamentos, técnicas e intervenções dirigidas a esta população específica, o que facilitaria a detecção precoce do problema, seu tratamento e seguimento ${ }^{22}$.

O tipo de bebida e as unidades de álcool ingeridas semanalmente permeiam a progressão dos problemas associados e a motivação para o tratamento. Altos índices de usuários de álcool vivendo sozinhos ou separados são congruentes com a evolução do que culmina no rompimento de laços familiares. A baixa escolaridade traduz pouca qualificação profissional, menos oportunidades e maior índice de desemprego. Para ambos os grupos, a amostra foi composta, em sua maioria, de pacientes com ensino fundamental incompleto, solteiras e desempregadas, congruentes com os achados de Wu e Ringwalt ${ }^{23}$, no entanto, as mudanças nos papéis do gênero, maior nível de escolaridade, independência financeira e trabalho fora de casa poderiam estar relacionadas ao aumento do consumo alcoólico entre mulheres ${ }^{24}$. Em estudo de Weisner ${ }^{25}$, observou-se que mulheres apresentaram menos apoio da família ou amigos para iniciar um tratamento do que os homens e que perdas pessoais, sociais e profissionais foram preditivas para a entrada no tratamento. Cronkite e Moos $^{26}$ apontam que mulheres solteiras tendem a apresentar mais sucesso no tratamento do que as casadas.

O abandono do tratamento observado neste estudo é congruente com achados literários. Stark ${ }^{21}$ relatou que o problema do abandono de tratamento é usualmente visto no contexto de doenças crônicas e que exigem tratamento prolongado e não é raro encontrarmos taxas de abandono acima de 50\% no primeiro mês de tratamento.

Há discrepâncias em relação à raça encontradas na literatura e os estudos deveriam sistematicamente explorar as diferenças de raça/etnia nos tratamentos ambulatoriais, objetivando melhor compreensão da natureza e possíveis origens destas diferenças ${ }^{27}$.

Estudo americano com pré-adolescentes, sendo o número de homens equivalente ao de mulheres, mostrou que há diferença entre os gêneros e raça para o uso inicial de substâncias. $O$ grupo das mulheres asiáticas brancas foi menos propenso ao consumo de substâncias do que o grupo de homens brancos, enquanto o grupo das mulheres negras apresentou maior consumo de álcool, tabaco e maconha do que o grupo de homens negros ${ }^{28}$.

No Brasil, o consumo de cervejas aparece em primeiro lugar, com 54 I per capita/ano, seguidos da cachaça 12 I per

Tabela 3. Distribuição das variáveis relacionadas a tratamentos prévios aos quais as pacientes estudadas foram substituidas

\begin{tabular}{|c|c|c|c|c|c|c|c|}
\hline Variáveis & Abandono rápido & $(\%)$ & Abandono gradual & $(\%)$ & Total & $(\%)$ & $p$ \\
\hline \multicolumn{8}{|l|}{ Medicaçäo coadjuvante } \\
\hline Nenhuma & 89 & (88) & 36 & (40) & 125 & (65) & \multirow[t]{3}{*}{$<0,001$} \\
\hline Dissulfiram & 10 & (10) & 49 & (54) & 59 & (31) & \\
\hline Naltrexona & 2 & (2) & 6 & (7) & 8 & (4) & \\
\hline \multicolumn{8}{|c|}{ Atendimento psiquiátrico na UNIAD } \\
\hline Não & 78 & (77) & 27 & (30) & 105 & (55) & \multirow[t]{2}{*}{$<0,001$} \\
\hline Sim & 23 & (23) & 64 & (70) & 87 & (45) & \\
\hline \multicolumn{8}{|l|}{ Tratamentos prévios } \\
\hline Alcoólicos Anônimos & 4 & (5) & 9 & (13) & 13 & (8) & \multirow[t]{8}{*}{0,028} \\
\hline Médico & 1 & (1) & 5 & (7) & 6 & (4) & \\
\hline Psicológico & 2 & (2) & 3 & (4) & 5 & (3) & \\
\hline Psiquiátrico & 18 & (21) & 8 & (11) & 26 & (16) & \\
\hline Ambulatorial & 2 & (2) & 6 & (8) & 8 & (5) & \\
\hline Outros & 3 & (3) & 2 & (3) & 5 & (3) & \\
\hline Nenhum tratamento & 57 & (66) & 38 & (54) & 95 & $(60)$ & \\
\hline Terapias combinadas & 14 & $(16)$ & 20 & (28) & 34 & (22) & \\
\hline
\end{tabular}


capita/ano e do vinho 1,8 I per capita/ano ${ }^{29}$. Observamos que, neste estudo, as mulheres do grupo "abandono gradual" consumiram mais destilados, enquanto as do grupo "abandono rápido" usaram mais fermentados ou a associação de fermentados e destilados.

Mulheres iniciam o consumo alcoólico mais tardiamente do que os homens, porém os problemas físicos aparecem mais cedo. Estudo de Kreutzberg ${ }^{30}$ mostrou que as mulheres apresentaram sua primeira internação psiquiátrica quatro anos e os homens oito anos após o início do consumo regular do álcool. A amostra em questão assemelha-se ao estudo de Borini ${ }^{31}$ quanto ao início do consumo alcoólico, idade das pacientes e quanto ao uso diário de bebidas alcoólicas.

A literatura aponta a existência de relação positiva entre o uso de medicações coadjuvantes e a continuidade do tratamento 32 . Em nosso estudo, o grupo "abandono gradual" iniciou o uso de dissulfiram ou naltrexona durante o decurso do tratamento e, ainda, o uso de coadjuvante apresentou significância estatística para a sua continuidade. O tratamento do alcoolismo não deve restringir-se à farmacologia, mas, sim, deve estar associado a outras modalidades de intervenções terapêuticas, como o aconselhamento às intervenções breves, as terapias comportamentais e que, apesar de a elevação do custo dos tratamentos adicionais, há benefício nos resultados terapêuticos.

Este estudo se propôs a caracterizar as mulheres do ambulatório da UNIAD, com abuso ou dependência de álcool, a partir dos prontuários, no período de 2000 a 2006. Há limitações neste estudo, pois se trata de estudo retrospectivo, com base apenas no prontuário das pacientes, o qual apresenta poucos instrumentos que poderiam mensurar e aprofundar a problemática. No entanto, o registro das variáveis estudadas e a entrevista realizada nesta população foram feitos pela mesma profissional, o que poderia diminuir a chance de vieses.

Algumas questões relevantes foram pouco exploradas, como as comorbidades psiquiátricas associadas ao beber, no entanto, as variáveis podem ser mais bem analisadas, o que contribuiria para melhor entendimento da população do estudo.

Há necessidade de novos estudos, como o seguimento das pacientes, a fim de identificar os motivos intrínsecos e extrínsecos relacionados com o abandono rápido do tratamento e intervenções estruturadas para esta população específica.

\section{CONCLUSÕES}

As mulheres com abuso ou dependência de álcool do grupo "abandono rápido" comparativamente ao grupo "abandono gradual" foram similares quanto às variáveis sociodemográficas, ao grau de dependência do álcool, à idade do início e freqüência do consumo alcoólico. Assemelharam-se quanto ao número de internações psiquiátricas prévias, utilizaram menos unidades de álcool/ semana e consumiram mais fermentados ou a associação destes com destilados. Não utilizaram ou fizeram menos uso de medicações coadjuvantes para o tratamento do alcoolismo, receberam menos atendimento psiquiátrico na UNIAD e assemelharam-se quanto ao número de internações psiquiátricas prévias. Diante destes resultados, concluímos que se faz necessário planejar estratégias de aderência ao tratamento específico para mulheres usuárias de álcool com a finalidade de redução deste agravo à saúde nesta população de pacientes.

\section{REFERÊNCIAS}

1. Meloni JN, Laranjeira R. Custo social e de saúde do consumo do álcool. Rev Bras Psiquiatr. 2004;26(1):7-10

2. Sjögren H, Eriksson A, Broström G, et al. Quantification of alcohol-related mortality in Sweden. Alcohol \& Alcoholism. 2000;35:601-11.

3. Wetterling T, Backhaus J, Junghanns K. Sucht im alter ein unterschätztes problem in der klinischen versorgung älterer menschen? Nervenarzt. 2002;73(9):861-6.

4. Marin-Leon L, Oliveira HB, Botega NJ. Alcoholism dependence mortality in Brazil: 19982002. Psicol Estud. 2007;12(1):115-121-4.

5. Pala B. II consumo alcolico femminile tra ricerca di parità e aumento del rischio: quale prevenzione? Ann Ist Super Sanità. 2004;40(1)411-46.

6. Hernandéz-Ávila CA, Rounsaville BJ, Kranzler HR. Opioid, cannabis and alcohol dependent women show more rapid progression to substance abuse treatment. Drug Alcohol Depend. 2004;74:265-72.

7. Nace EP, Saxen JJ, Shore N. Borderline disorders in alcoholic female psychiatric patients: a case control study. Psychopatology. 1985;18:50-60.

8. Rudolf $\mathrm{H}$, Priebe S. Subjetive quality of life and symptoms in women with alcoholism during detoxification treatment. Drug Alcohol Depend. 2002;66(1):71-6.

9. Frye MA, Altshuler LL, Mc Elroy SL, Suppest T. Gender differences in prevalence, risk and clinical correlates of alcoholism comorbity in bipolar disorder. Am J Psychiatric. 2003; 160(5):883-9.

10. Kinrys G, Wygant LE. Anxiety disorders in women: does gender matter to treatment? Rev Bras Psiq. 2005;27 Suppl(I):43-50.

11. Goldblum DS, Naranjo CA, Bremer KE, et al. Eating disorders and alcohol abuse in women. British J Addiction. 1992;87:913-20.

12. Blume SB, Zilberman ML. Women: clinical aspects. In: Lowison J, Ruiz P, Millman RB, et al., editores. Substance abuse: a comprehensive textbook. $4^{\text {th }}$ ed. Lippincott Willians \& Wilkins; 2004. p. 1049-64.

13. Méier PS, Donmall MC, McElduff $P$, et al. The role of the early therapeutic alliance in predicting drug treatment dropout. Drug Alcohol Depend. 2006;83;57-64.

14. Greenfield SF, Brooks AJ, Gordon SM, et al. Substance abuse treatment entry, retention and outcome in women: a review of the literature. Drug Alcohol Depend. 2006:1-21.

15. Siqueira MM, Garcia MLT, Souza RS. 0 impacto das faltas às consultas em um programa de dependentes de álcool. J Bras Psiquiatr. 2005;54(2):114-9.

16. Rennó Jr J, Fernandes CE, Mantese JC, et al. Saúde mental da mulher no Brasil: desafios clínicos e perspectivas em pesquisa. Rev Bras Psiquiatr. 2005;27(2):73-6.

17. Baekland F, Lundwall L. Dropping out of treatment: a critical review. Psychological Bulletin. 1975;82(5):738-83.

18. Miller WR, Rollnick S. Motivacional interviewing-preparing people to change addictive behavior. New York: Guilford Press; 1991 
19. Jorge MR, Masur J. The use of the short-form alcohol dependence data questionnaire (SADD) in Brazilian alcoholic patients. British Journal of Addiction. 1985;80:301-5.

20. Pillon SC, Nóbrega MPSS. Desintoxicação al coólica ambulatorial realizada por enfermeiras. In: Focchi GA, Leite MC, Laranjeira R, Andrade AG. Dependência química: novos modelos de tratamento. São Paulo: Roca; 2001. p. 143-60.

21. Stark MJ. Dropping out of substance abuse treatment: a clinically oriented review. Clin Psychol Rev. 1992;12:93-116

22. Pillon SC, Laranjeira R. Formal education and nurses' attitudes towards alcohol and alcoholism in a Brazilian sample. São Paulo Med J. 2005;123(4):175-80.

23. Wu LT, Ringwalt CL. Alcohol dependence and use of treatment services among women in the community. Am J Psychiatry. 2004;161:1790-7.

24. Fillmore KM. When angels fall: women's drinking as cultural preoccupation and as reality. In: Wilsnack RW, Vogeltanz ND, Wilnack SC, Harris R. Gender differences in alcohol consumption and adverse drinking consequences: cross-cultural patterns. Addiction. 2000;95(2):251-65.

25. Weisner $C$. Toward an alcohol treatment entry model: a comparison of problem drinkers in the general population and in treatment. Alcohol Clin Exp Res. 1993;17:746-52.
26. Cronkite RC, Moos RH. Sex and marital status in relation to the treatment an outcome of alcoholic patients. Sex Roles. 1984;11:93-112.

27. Johnson P, Richter L, Herbert KD, McLellan AT, Carise DA. Telescoping of drinking-related behaviors: gender, racial/ethnic and age comparisions. Substance Use Misuse. 2005; 40(8):1139-51.

28. Gillmore MR, Catalano RF, Morrison DM, et al. Racial differences in acceptability and availability of drugs and early iniciation of substance use. Am J Drug Alcohol Abuse. 1990;16(3-4):185-206.

29. Galduróz JC, Caetano R. Epidemiologia do uso de álcool no Brasil. Rev Bras Psiquiatr. 2004;26(1):3-6.

30. Kreutzberg K. Frauen leiden meist starker unter dem Alkoholismus. Fortschritte der Medizin. 1998;16(24):43-4.

31. Borini P, Soi EA Rubira KP, et al. Alcoolismo feminino: padrão de consumo, motivações para 0 abuso e aspectos conceituais e emocionais de pacientes de baixa renda internadas em hospital psiquiátrico. J Bras Psiquiatr. 1999;(12):539-45.

32. Kranzler HR. Pharmacotherapy of alcoholism: gaps in knowledge and opportunities for research. Alcohol Alcohol. 2000;35(6):537-47. 